\title{
Metastatic Renal Cell Carcinoma Mimicking Trigeminal Schwannoma in a Patient Presenting with Trigeminal Neuralgia
}

\author{
Arthur Wang ${ }^{1}$ George Kleinman ${ }^{2}$ Raj Murali ${ }^{1}$ John Wainwright ${ }^{1}$ Adesh Tandon ${ }^{1}$ \\ ${ }^{1}$ Department of Neurosurgery, New York Medical College, \\ Westchester, Valhalla, New York, United States \\ 2 Department of Pathology, New York Medical College, Westchester, \\ Valhalla, New York, United States \\ Address for correspondence Arthur Wang, MD, Department of \\ Neurosurgery, New York Medical College, 100 Woods Road, Valhalla, \\ NY 10595, United States (e-mail: wanga@wcmc.com).
}

J Neurol Surg Rep 2015;76:e282-e286.

\begin{abstract}
We present an unusual case of a metastatic renal cell carcinoma (RCC) mimicking trigeminal schwannoma. The patient, with no prior history of RCC, presented with clinical symptoms and imaging consistent with trigeminal neuralgia secondary to

Keywords

- metastatic renal cancer

- renal cell carcinoma

- trigeminal schwannoma

- trigeminal neuralgia

- cerebellopontine angle trigeminal schwannoma. Magnetic resonance imaging of the brain showed a large bilobed cystic/solid mass primarily in the cerebellopontine angle cistern, with extension into the left middle cranial fossa, Meckel cave, and left cavernous sinus. Following surgical excision, histopathology revealed the tumor to be an RCC infiltrating into the trigeminal nerve fascicles. Further imaging and investigation revealed widespread metastasis to the vertebral bodies and long bones. Metastatic RCC to the trigeminal nerve is rare. Despite the development of more effective treatment modalities, the prognosis of metastatic RCC remains poor. To our knowledge, this is the first reported case of RCC metastasizing to the trigeminal nerve fascicles.
\end{abstract}

\section{Introduction}

Approximately 8 to $10 \%$ of all intracranial tumors arise in the cerebellopontine angle (CPA). ${ }^{1}$ The vast majority of CPA tumors are vestibular schwannomas followed by meningiomas and epidermoid tumors. About $0.2 \%$ of all lesions found in the CPA are metastatic in origin. ${ }^{1}$ Common sources include primary neoplasms of the lung, breast, prostate, nasopharynx, and oropharynx. ${ }^{2-5}$ Metastatic tumors in this location present with rapidly progressive lower cranial nerve symptoms such as facial nerve palsies. ${ }^{4}$ We present the case of a patient presenting with symptoms of trigeminal neuralgia and a skull base lesion that upon surgical excision proved to be metastatic renal cell carcinoma (RCC).

received

May 26, 2015

accepted after revision

August 19, 2015

published online

October 29, 2015

\section{Case Report}

\section{History and Examination}

A 54-year-old man with no significant past medical history presented to the office with a 1-year history of mild headaches, recurrent episodes of sharp left facial pain, and progressive left facial numbness. He described the sharp pain as an electrical shock over his left eye and cheek (V1, V2) that was triggered by chewing, brushing his teeth, and washing his face. The pain lasted from a few seconds to a minute and had been increasing in frequency over the past few months. On neurologic examination, he was noted to have hypoesthesia to light touch and pinprick in the left $\mathrm{V} 1,2,3$ distributions. There was no motor weakness in the

\footnotetext{
(c) 2015 Georg Thieme Verlag KG Stuttgart · New York
} 
muscles of mastication. The remainder of the examination was unremarkable.

Magnetic resonance (MR) imaging revealed a heterogeneous partially solid and partially cystic bilobed enhancing mass in the left CPA cistern extending ventrally into the medial left middle cranial fossa and into the left Meckel cave and cavernous sinus ( $\mathbf{- F i g}$. 1). The mass appeared to course along the left trigeminal nerve and compressed the adjacent left pons and middle cerebellar peduncle. Preoperative radiologic findings and clinical history were compatible with trigeminal schwannoma (TS).

\section{Operation}

Given that the lesion was symptomatic and showed brainstem compression, the decision was made to proceed with surgical excision of the lesion. A left subtemporal approach was undertaken to explore the entire mass. On opening, we found tumor in the tentorial incisura. The tentorium was then opened parallel to the superior petrosal sinus exposing the tumor in the CPA. We found a highly vascular tumor extending through the porus trigeminy into the cavernous sinus and extending through Meckel cave underneath the tentorium into the posterior fossa causing brainstem compression. The lesion was closely admixed with fascicles of the trigeminal nerve. A large amount of tumor was resected in the Meckel cave, middle cranial fossa, and cavernous sinus. The tumor specimen was sent for pathologic analysis.

\section{Pathologic Findings}

Histopathologic examination revealed the tumor to be a carcinoma. Microscopy of the tumor revealed trigeminal nerve fascicles with infiltrating tumor cells $(\boldsymbol{- F i g}$. $2 \mathbf{2 A}$ ) that were polygonal in shape with clear cytoplasm ( - Fig. 2B). The tumor cells were negative for neurofilament, whereas the nerve fibers were stained positively for neurofilament (-Fig. 3A). Myelinated nerve fibers that stained positive for myelin basic protein were seen infiltrated by surrounding tumor cells (-Fig. 3B). The tumor cells stained positive for the immunohistochemical stains PAX8 and CA9 consistent with a renal origin (-Fig. 3C, D). Taken together, these findings are consistent with an RCC metastasis to the trigeminal nerve.

\section{Postoperative Course}

The patient did well after the surgery, and the postoperative neurologic assessment showed a transient left facial nerve palsy. Computed tomography (CT) and MR imaging after surgery revealed no evident residual tumor. After learning the diagnosis, a metastatic work-up was undertaken. Further imaging revealed multiple renal lesions bilaterally as well as lytic lesions of his vertebral bodies, calvarium, and long bones. The patient was planned for chemotherapy for his RCC but left against medical advice. Three months later, the patient presented for pneumonia, and a follow-up MR imaging of the brain was obtained demonstrating no recurrent tumor.

\section{Discussion}

Intracranial metastases are the most common intracranial neoplasm with a yearly incidence of 200,000 cases. ${ }^{6}$ Metastasis to the skull base presents with five characteristic syndromes $^{7}$ : (1) orbital syndrome, consisting of exophthalmos and extraocular paresis; (2) parasellar syndrome,
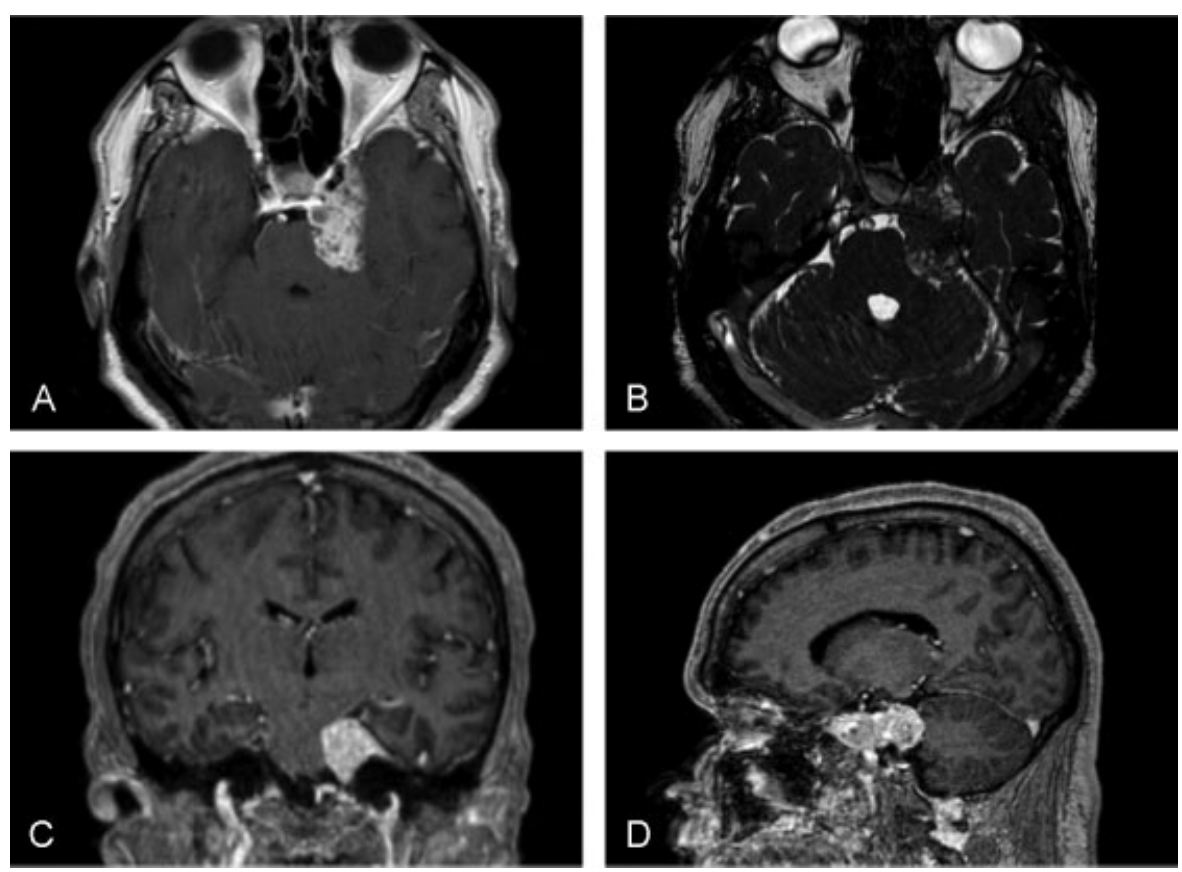

Fig. 1 Preoperative magnetic resonance imaging (MRI) of the ectopic renal cell carcinoma. (A, B) Axial T1-weighted and T2-weighted MRI sequences demonstrating a heterogenous, partially solid, and cystic enhancing mass in the left cerebellopontine angle cistern extending ventrally into the medial left middle cranial fossa and into left Meckel cave and left cavernous sinus. (C, D) Coronal and sagittal T1-weighted MRI sequences show the lesion compressing the adjacent left pons and left middle cerebellar peduncle. There is partial encasement of the cavernous left internal carotid artery from the mass lesion. 

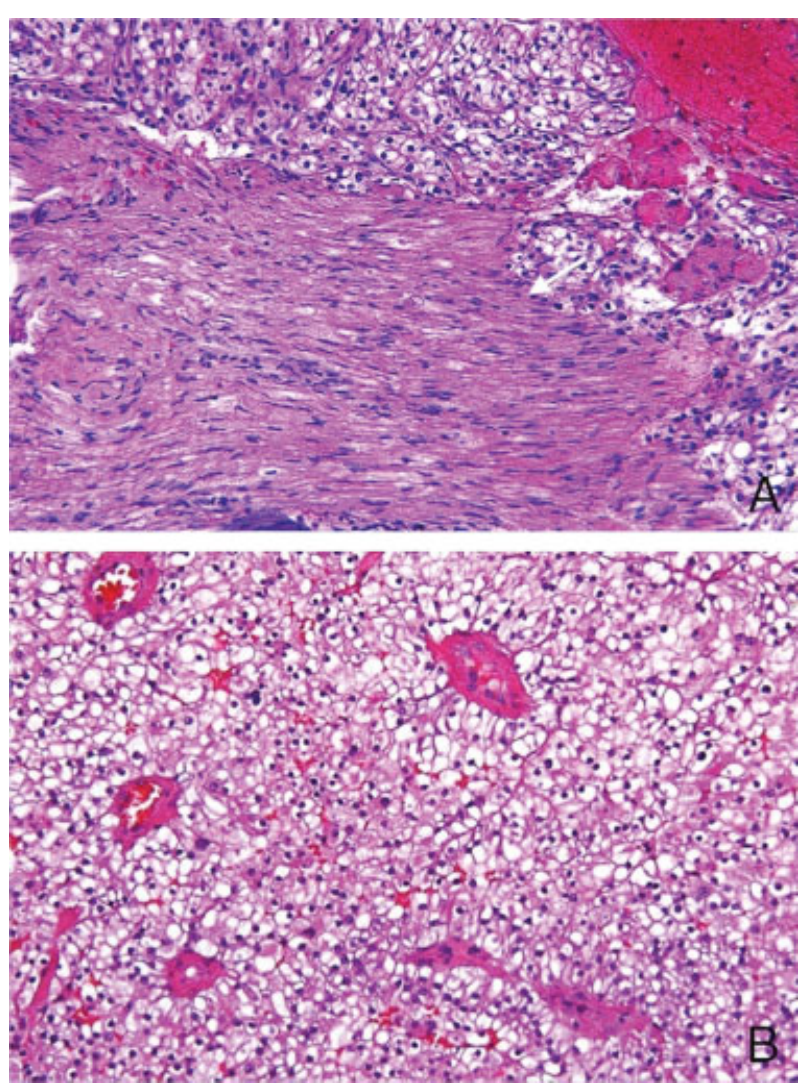

Fig. 2 Light photomicrographs of the surgical specimen (hematoxylin and eosin). (A) The tumor cells are invading the nerve (arrow). (B) High magnification show large polygonal cells with clear cytoplasm and uniform round nuclei but irregular contours.

producing ocular paresis without proptosis; (3) middle fossa syndrome, causing facial numbness/pain and sensory loss in the trigeminal nerve distribution; (4) jugular foramen syndrome, consisting of unilateral occipital pain and evidence of lower cranial nerve dysfunction; and (5) occipital condyle syndrome, consisting of occipital pain, neck stiffness, and hypoglossal nerve palsy.

Our patient presented with symptoms of classic trigeminal neuralgia. MRI showed an enhancing dumbbell-shaped tumor that followed the course of the trigeminal nerve into the Meckel cave. The radiographic appearance was most consistent with a bicompartmental TS that originated in the Meckel cave and extended into the posterior fossa through the porus trigeminus. About $20 \%$ of TS have a dumbbell shape, extending from the middle to the posterior cranial fossa. ${ }^{8,9}$ The differential diagnosis of a Meckel cave TS are CPA meningioma and metastasis, although their incidence is less frequent than that of a TS. The most common presenting symptom of TS is decreased facial sensation followed by trigeminal neuralgia and trigeminal motor weakness. ${ }^{9}$ Thus differentiating meningiomas and metastasis from TS can be difficult.

Surgery via a subtemporal approach revealed a highly vascular tumor admixed with fibers of the trigeminal nerve. Histologic examination demonstrated a CPA RCC with infiltration into the nerve fibers consistent with the intraoperative findings of a tumor intermingled with the fascicles of the trigeminal nerve. Previous studies have reported focal metastasis of the trigeminal nerve from metastatic breast carcinoma, melanoma, lung carcinoma, esophageal adenocarcinoma, and lymphoma. Our case demonstrates a focal intracranial metastasis from a RCC to the trigeminal nerve on histopathology. Solitary metastasis to the trigeminal nerve via the hematogenous route is rare in the literature. The vascular supply to the trigeminal nerve root is from the trigeminal arteries of the superolateral pontine artery and peduncular cerebellar artery. These arteries supply surrounding dura and brain parenchyma so if metastasis occurs within the nerve, the surrounding brain tissue will also have already been infiltrated. ${ }^{9}$

RCC comprises 3\% of adult malignancies and the third most frequent carcinoma with metastasis to the head and neck region, preceded by breast and lung cancer. ${ }^{5}$ Overall, $85 \%$ of renal cancers are the clear cell variety. Because the kidneys receive a quarter of the circulating blood volume, RCC has a high potential for hematogenous spread. Additionally, tumor emboli bypass the pulmonary filters by traveling in the Batson venous plexus between the valveless paravertebral and epidural venous systems. ${ }^{2,3}$

The cerebellopontine cistern is a rare location for metastases to occur. Only $0.2 \%$ of intracranial tumors found in the CPA are metastatic in origin. ${ }^{1,10}$ RCCs have an $11 \%$ incidence of brain metastases representing $\sim 1,100$ patients per year. ${ }^{11} \mathrm{~A}$ literature search revealed nine case reports of metastatic RCC presenting within the skull base and five case reports presenting within the sinonasal cavity. ${ }^{2,3,7,12-16}$ of the nine cases presenting within the skull base, only three presented exclusively within the CPA. 2,4,17 Many of the patients described in these reports had a previous history of malignancy. However, as our case report shows, this is not always the case, and a cerebellopontine lesion can be the first presentation of a malignancy.

Current treatment options in patients with metastatic RCC to the brain include surgical resection, corticosteroid therapy, and Gamma Knife surgery. Because of the relative radioresistance of RCC to traditional fractionated radiation delivery, radiation therapy and/or radiosurgery in combination with resection and systemic chemotherapy are the current treatment algorithms. ${ }^{3,10,11,18-20}$ Shuto et al demonstrated the effectiveness of GKS with a dose $\geq 20 \mathrm{~Gy}$ for the treatment of metastatic brain tumors from $\mathrm{RCC}<3 \mathrm{~cm}$ in maximum diameter. ${ }^{21}$ Additionally, adjuvant chemotherapeutic drugs targeting tyrosine kinase receptors such as sorafenib and sunitinib show promise as second-line agents for the treatment of metastatic RCC. ${ }^{6}$ Despite the development of more effective treatment modalities, the prognosis of metastatic RCC is poor. Excision of solitary RCC metastatic lesions following nephrectomy resulted in a 2-year survival rate of $41 \%$ and a 5 -year survival rate of $13 \%{ }^{5}$ Benanni et al showed that patients with intracranial metastasis from RCC had a median survival of 13.3 months upon discovery of their brain metastasis and identified three significant factors associated 

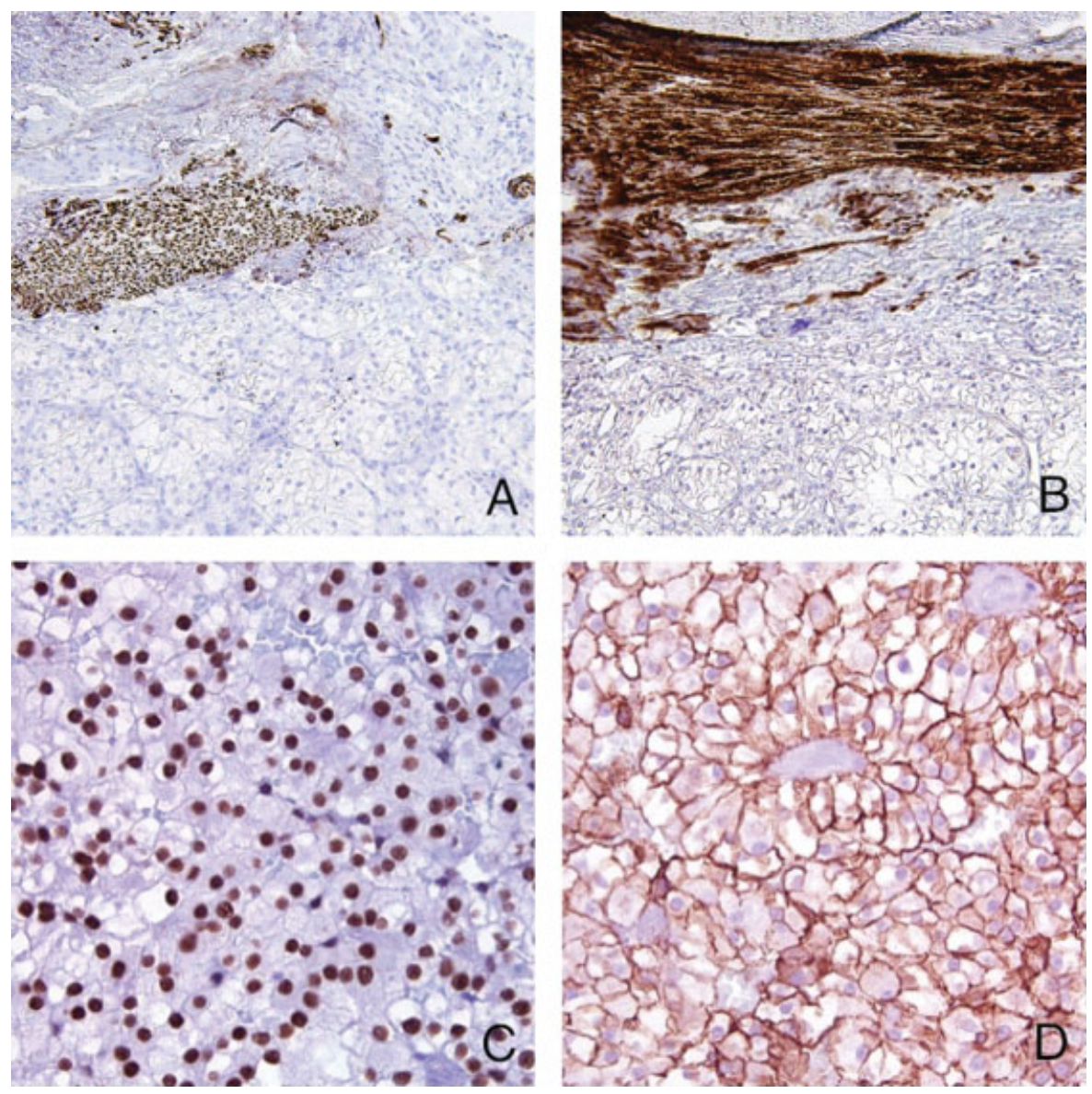

Fig. 3 Photomicrographs of tumor sections with immunohistochemical staining. (A) The tumor cells are negative for neurofilament, whereas the nerve fibers are stained positively. (B) Myelinated nerve fibers that stain positive for myelin basic protein are infiltrated by surrounding tumor cells. (C, D) Immunohistochemistry using antibodies to (C) PAX8 and (D) CA9 confirms a clear cell lesion with a renal origin.

with longer patient survival: absence of intracranial hypertension, superficial surgically accessible solitary lesions, and the absence of systemic metastasis. ${ }^{3}$

The present study is a case of a RCC metastasis to the CPA with infiltration into the trigeminal nerve that was verified surgically and histopathologically. Previous case reports included patients with an already established diagnosis of RCC prior to discovery of a metastatic lesion. Although there can be such a history of malignancy, it does not always have to be the case. Our case report shows that a trigeminal schwannoma-like lesion can be the first presentation of a malignancy. This case highlights the need to consider metastatic lesions in the differential diagnosis in these patients. A routine preoperative work-up with a CT scan of the chest, abdomen, and pelvis may prevent this pitfall.

\section{References}

1 Lalwani AK. Meningiomas, epidermoids, and other nonacoustic tumors of the cerebellopontine angle. Otolaryngol Clin North Am 1992;25(3):707-728

2 Bhatoe HS, Deshpande GU. Cerebellopontine angle metastasis from clear-cell renal carcinoma presenting as bleeding from the ear. J Laryngol Otol 1996;110(4):370-372
3 Bennani O, Derrey S, Langlois O, et al. Brain metastasis from renal cell carcinoma. Neurochirurgie 2014;60(1-2):12-16

4 Panarese A, Turner J, Fagan PA. Renal carcinoma metastasis: an unusual cerebellopontine angle tumor. Otolaryngol Head Neck Surg 2002;127(3):245-247

5 Sepúlveda I, Platin E, Klaassen R, et al. Skull base clear cell carcinoma, metastasis of renal primary tumor: a case report and literature review. Case Rep Oncol 2013;6(2):416-423

6 Hill KL Jr, Lipson AC, Sheehan JM. Brain magnetic resonance imaging changes after sorafenib and sunitinib chemotherapy in patients with advanced renal cell and breast carcinoma. J Neurosurg 2009;111(3):497-503

7 Endo K, Okano R, Kuroda Y, Yamada S, Tabei K. Renal cell carcinoma with skull base metastasis preceded by paraneoplastic signs in a chronic hemodialysis patient. Intern Med 2001;40(9):924-930

8 De Pena CA, Lee YY, Van Tassel P. Lymphomatous involvement of the trigeminal nerve and Meckel cave: CT and MR appearance. AJNR Am J Neuroradiol 1989;10(5, Suppl):S15-S17

9 Nakano I, Iwasaki K, Kondo A. Solitary metastatic breast carcinoma in a trigeminal nerve mimicking a trigeminal neurinoma. Case report. J Neurosurg 1996;85(4):677-680

10 Kotecha R, Angelov L, Barnett GH, et al. Calvarial and skull base metastases: expanding the clinical utility of Gamma Knife surgery. J Neurosurg 2014;121(Suppl):91-101

11 Payne BR, Prasad D, Szeifert G, Steiner M, Steiner L. Gamma surgery for intracranial metastases from renal cell carcinoma. J Neurosurg 2000;92(5):760-765 
12 Brener ZZ, Zhuravenko I, Jacob CE, Bergman M. An unusual presentation of renal cell carcinoma with late metastases to the small intestine, thyroid gland, nose and skull base. Nephrol Dial Transplant 2007;22(3):930-932

13 Mendelson ZS, Patel AA, Eloy JA, Liu JK. Endoscopic palliative decompression of the cavernous sinus in a rare case of a metastatic renal cell carcinoma to the clivus. Br J Neurosurg 2015;29(3): 430-431

14 Parida PK. Renal cell carcinoma metastatic to the sinonasal region: three case reports with a review of the literature. Ear Nose Throat J 2012;91(11):E11-E16

15 Remenschneider AK, Sadow PM, Lin DT, Gray ST. Metastatic renal cell carcinoma to the sinonasal cavity: a case series. J Neurol Surg Rep 2013;74(2):67-72

16 Streitmann MJ, Sismanis A. Metastatic carcinoma of the temporal bone. Am J Otol 1996;17(5):780-783
17 Mubaidin SI, Sunna JB, Beiruti MA, Shennak MM, Ayoub MS. Renal cell carcinoma presenting as Garcin's syndrome. J Neurol Neurosurg Psychiatry 1990;53(7):613-614

18 Chamoun RB, Suki D, DeMonte F. Surgical management of cranial base metastases. Neurosurgery 2012;70(4):802-809; discussion 809-810

19 Kim CH, Chung SK, Dhong HJ, Lee JI. Cerebrospinal fluid leakage after gamma knife radiosurgery for skull base metastasis from renal cell carcinoma: a case report. Laryngoscope 2008;118(11): 1925-1927

20 Maxwell M, Borges LF, Zervas NT. Renal cell carcinoma: a rare source of cauda equina metastasis. Case report. J Neurosurg 1999; 90(1, Suppl):129-132

21 Shuto T, Inomori S, Fujino H, Nagano H. Gamma knife surgery for metastatic brain tumors from renal cell carcinoma. J Neurosurg 2006;105(4):555-560 